\title{
EXTENSION PROPERTIES OF BANACH SPACES
}

\author{
BY ANDREW SOBCZYK ${ }^{1}$ \\ Communicated by F. Bohnenblust, January 16, 1962
}

A bounded linear transformation $a$ on a Banach space $L$, to a Banach space $M$, is called regular (see [9, Part II, Chapter 2; 2, pp. 25-26]), in case there is a bounded linear transformation $x$ on $M$ to $L$, such that $a x a=a$. The transformation $a$ is possibly regular only if it is of type IIA $[13 ; 14]$, that is only if the null space $\Re(a)$ of $a$ has a closed complement $\mathfrak{T}^{c}$, and if $a$ restricted to $\Re^{c}$ is an isomorphism $g$ of $\mathscr{T}^{c}$ onto the range $R(a)$ of $a$. For such an $a$, the projection $p$ through $\mathscr{T}(a)$ onto $\mathscr{N}^{c}$ is bounded. Then in order that $a x a=a$, it is necessary and sufficient that $x$ be a bounded linear extension to $M$ of the inverse isomorphism $g^{-1}$. In this case clearly $x a$ is the bounded projection $p$ of $L$ through $\Re(a)$ onto $\Re^{c}$, and $a x$ is a bounded projection $q$ of $M$ onto $R(a)$. Therefore even if $a$ is of type IIA so that the bounded projection $p$ exists, the transformation $a$ cannot be regular if there is no bounded projection $q$ of $M$ onto $R(a)$. In case the extension $x$ exists so that $a$ is regular, the transformation $b=p x=x a x$ on $M$ to $L$ is a relative inverse [2] of $a$; that is $a b a=a$ and $b a b=b$. In case $L$ and $M$ are Hilbert spaces, $g$ is isometric, and $p$ and $q$ are orthogonal projections, the relative inverse $b$ coincides with the adjoint $a^{*}$ of $a$. (Every bounded linear transformation on a Hilbert space to a Hilbert space necessarily is of type IA or IIA. ${ }^{2}$ )

For reasons including those suggested in the preceding paragraph, relations between various properties concerning projections and extensions in Banach spaces are of interest. In this note the equivalence of several such properties, which are seemingly different, is established. The main result is the equivalence of the extension property to the uniform extension property. (See the next sections for definitions.) This is a consequence of the following theorem.

THEOREM 1. If a Banach space $B$ has the property that there is a

\footnotetext{
1 This research was supported under Contract No. AF 49 (638)-1055, by the Air Force Office of Scientific Research.

${ }^{2}$ For suppose that $a$ is bounded on a Hilbert space $L$ to a Hilbert space $M$. In order that $a$ be of type IB or IIB, it would be necessary that every complement of $\mathscr{N}(a)$ be not closed, but the orthogonal complement is closed for any $\mathscr{N}(a)$. In case $a$ is not of type IIA, then neither $\mathcal{R}(a)$ nor $\mathcal{R}\left(a^{*}\right)$ is closed, and it follows from $(a x, y)$ $=\left(x, a^{*} y\right)$ that the closure of $\mathscr{R}(a)$ is the orthogonal çomplement of $\mathscr{T}\left(a^{*}\right)$, and that the closure of $\mathcal{R}\left(a^{*}\right)$ is the orthogonal complement of $\mathscr{T}(a)$. An $a$ of type IA is not regular, and its adjoint $a^{*}$ is not a relative inverse of $a$.
} 
bounded projection of $C$ onto $B$, for every Banach space $C$ which is a superspace of $B$, then there exists a constant $K=K(B)$, independent of $C$, such that for each superspace $C$, there is a projection onto $B$ having bound less than or equal to $K$.

1. Projection and extension properties. A Banach space $L$ will be said to have the extension property if each bounded linear transformation $u$ from any linear subspace $R$ of any Banach space $M$, into $L$, has a continuous linear extension $U$ on $M$ with range in $L$. A Banach space $B$ has the projection property in case for any superspace $C$ containing $B$ which is also a Banach space, there exists a bounded projection of $C$ onto $B$. A finite dimensional space $L$, for example, has both the extension and the projection properties.

For regularity of $a$, extensibility only of the isomorphism $g^{-1}$ is required. A Banach space $L$ has the extension property for isomorphisms into in case each isomorphism $u$ of a subspace $R$ of $M$ into $L$ can be extended to be a bounded linear transformation on $M$ into $L$. If $M$ is a copy of $L$, of course the identity on $R$ to $R$ can be extended to be the identity on $L$ to $L$.

THEOREM 2. For any Banach space B, the extension property for isomorphisms into, the projection property, and the extension property, are all equivalent; i.e. if $B$ has any one of the properties, it has all three.

Proof. If $u$ is the identity on $B \subset C$ to a copy of $B$, then the extension $U$ to $C$ of $u$ is a projection of $C$ onto $B$; thus the extension property for isomorphisms into implies the projection property.

An extension theorem of [13] states that for any bounded linear transformation on a subspace, there always exists a bound-preserving extension if enlargement of the range is permitted. By that theorem, $u$ on $R \subset M$ to $B$ has an extension $V$ on $M$ to $C \supset B$ with $|V|=|u|$; if $p$ is a continuous projection of $C$ onto $B$, then $U=p V$ is an extension of $u$ with bound not exceeding $|p| \cdot|u|$. Therefore the projection property implies the extension property. Finally, the extension property in particular implies the extension property for isomorphisms. Therefore all three properties are equivalent.

We note the following property, which is a consequence of the discussion of regular elements $a$, for a Banach space $B$ which has the extension property: The existence or nonexistence of a continuous projection of $B$ onto a linear subspace $D$ is independent of the particular imbedding of $D$ in $B$; i.e. if $D_{1} \subset B$ is any subspace isomorphic with $D$, then there is a continuous projection of $B$ onto $D_{1}$ if and only if there is one onto $D$. 
THEOREM 3. If a Banach space $B$ has the extension property, and if there is a continuous projection of $B$ onto a linear subspace $D$, then $D$ has the extension property. If $B=B_{1} \oplus B_{2}$, where $B_{1}$ and $B_{2}$ both have the extension property, then $B$ has the extension property.

Proof. Let $u$ be a bounded linear transformation on a subspace $R \subset M$ to $D \subset B$, and $p$ the projection of $B$ onto $D$. Since $B$ has the extension property, there is a bounded extension $V$ with range in $B$. Then $U=p V$ is the required continuous linear extension with range in $D$.

If $B=B_{1} \oplus B_{2}$, then $u \xi=u_{1} \xi+u_{2} \xi, \quad u_{1} \xi \in B_{1}, u_{2} \xi \in B_{2}$. We have $\left|U_{1} \xi+U_{2} \xi\right| \leqq\left(\left|U_{1}\right|+\left|U_{2}\right|\right)|\xi|$, where $U_{1}$ is the continuous extension with range in $B_{1}$ of $u_{1}$, and $U_{2}$ is the continuous extension with range in $B_{2}$ of $u_{2}$. Therefore $U=U_{1}+U_{2}$ is a continuous extension of $u$, with range in $B$, as required.

Theorems 2 and 3 transform a question of the author (in [11]), of whether there exists any separable, infinite-dimensional subspace $W$ of the Banach space $(m)$ of all bounded sequences, which has a continuous projection from $(m)$, into the following: Does there exist any separable, infinite-dimensional Banach space $W$ which has the extension property? Such a space $W$ could be imbedded in the space $(C)$ of continuous functions on a closed interval, which in turn is imbedded in $(m)$. (As shown in [11], the separable spaces $\left(c_{0}\right)$ and $(C)$ do not have the extension property.) The result of [6] suggests the questions: Does there exist a separable, infinite-dimensional space of continuous functions on an extremally disconnected compact Hausdorff space? An affirmative answer to this would provide an example of a space $W$. Is any $W$ necessarily isomorphic to such a space? Is there any infinite-dimensional subspace of $\left(c_{0}\right)$ which has the extension property? (It is shown in [12] that $\left(c_{0}\right)$ contains a subspace which has no continuous projection from $\left(c_{0}\right)$.) Is the intersection of all subspaces of $(m)$, which contain $\left(c_{0}\right)$ and have the extension property, a space which has the extension property? (Conceivably it might be $\left(c_{0}\right)$, or a subspace $Z \supset\left(c_{0}\right)$, such that there is a continuous projection of $Z$ onto $\left(c_{0}\right)$-in that case, by Theorem 3 the space $Z$ does not have the extension property.)

A linear space $L$, such that each IIA bounded linear transformation $a$ of $L$ into itself is regular, must have the property: (1) that each isomorphism $x$ to $L$, from a subspace of $L$ which is a possible range $\mathcal{R}(a)$, can be extended to be a continuous linear transformation on $L$ to $L$. Hilbert space has property (1), which is weaker than the extension property. Property (1) of $L$ evidently is equivalent to each of the following: (2) every IIA bounded linear transformation on $L$ to 
$L$ is regular, (3) if a subspace of $L$ has a continuous projection from $L$ in one imbedding, it has a continuous projection from $L$ in any other imbedding. (In particular, if $L$ is isomorphic with a proper subspace, there must exist a continuous projection of $L$ onto the subspace.)

2. Uniform properties. A Banach space $L$ has the uniform extension property with bound $K$, in case it has the extension property and each bounded linear transformation $u$ from a subspace $R$ into $L$ can be extended to the whole space $M$, with multiplication of the bound of $u$ by not more than $K_{1}, K=\inf K_{1}$; i.e. for arbitrary $\epsilon>0$, extension is always possible with multiplication of the bound by not more than $(K+\epsilon)$. (See [1].) Similarly a Banach space $B$ has the uniform projection property with bound $K$, if projection of $C$ onto $B$ is always possible, with bound not exceeding $K+\epsilon$, and $K=K(B)$ is the smallest possible number having this property for the Banach space $B$.

The following theorems for the uniform properties, which correspond to Theorems 2 and 3, are obtained by slight additions to the proofs of those theorems.

THEOREM 2a. If a Banach space B has the uniform extension property for isomorphisms into with bound $K_{2}$, the uniform projection property with bound $K$, and the uniform extension property with bound $K_{1}$, then $K_{2}=K=K_{1}$.

THEOREM 3a. If a Banach space B has the uniform extension property with bound $K$, and if there is a continuous projection $p$ of $B$ onto a linear subspace $D$, then $D$ has the uniform extension property with bound not exceeding $|p| \cdot K$. If $B=B_{1} \oplus B_{2}$, where $B_{1}, B_{2}$ have the uniform extension property with respective bounds $K_{1}, K_{2}$, then $B$ has the uniform extension property with bound not exceeding $K_{1}+K_{2}$.

A finite-dimensional example in [1] shows that if $k=\inf |p|$ for all continuous projections of $B$ onto $D$, the uniform bound $K_{3}$ for $D$ may be less than $k K$.

Banach spaces having the uniform extension property with bound 1 have been characterized $[4 ; 8 ; 6]$ as spaces of all continuous functions on an extremally disconnected compact Hausdorff space. The space $(m)$ of all bounded sequences, for example, has this property. Since also $(m)$ has no continuous projection onto its subspace $(c)$ of convergent sequences $[10 ; 11]$, there does not exist a bounded linear transformation of type IIA of $(m)$ onto any space $M$ which is isomorphic with all of $(c)$. Open questions: Does there exist any bounded linear transformation $a$ on $(m)$ to $(c)$, with $\mathcal{R}(a)=(c)$ ? Is each Banach 
space which has the uniform extension property with bound $K, K>1$, isomorphic with a Banach space which has the uniform extension property with bound 1 ?

3. Separable spaces. Similar properties may be defined for a separable Banach space $B$, in terms of Banach superspaces which are restricted to being separable.

Theorem 4. The Banach space $\left(c_{0}\right)$ has the separable uniform projection property with bound 2. The Banach space (c) has the separable uniform projection property with bound 3.

Proof. It is shown in [11] that for any separable subspace $W$ of $(m)$ which contains $\left(c_{0}\right)$, there is a projection of bound 2 of $W$ onto $\left(c_{0}\right)$, and in [7] that for any separable subspace $Z$ of $(m)$ which contains $(c)$, there is a projection of bound 3 of $Z$ onto $(c)$. Suppose that $B$ is any separable Banach superspace of say $(c)$. As in the discussion of regular elements, let $g$ be the isometry of $(c) \subset(m)$ onto $(c) \subset B$. Since $(m)$ has the uniform extension property with bound 1 , a boundpreserving extension $x$ of $g^{-1}$ to $B$ exists. The range $R(x) \subset(m)$ and its closure are separable since $B$ is separable. Therefore there is a projection $p=x a$ of bound not exceeding 3 of $R(x)$ onto $(c)$. Then $q=a x$ is the required projection of bound not exceeding 3 of $B$ onto (c). Since as shown in [11] and [7], the bounds 2 and 3 are the best possible, it follows that $(c)$ has the separable uniform extension property with bound 3 , and that $\left(c_{0}\right)$ has the separable uniform extension property with bound $2 .^{3}$

The subspaces of $\left(c_{0}\right)$ and $(c)$, for which there is no continuous projection, as given in $[12]$, do not have the separable extension property.

The space $C(E)$ of continuous functions on a compact Hausdorff space $E$ is separable if and only if $E$ is metrizable [15, p. 245]. Therefore the question of whether there is a separable Banach space which has the uniform extension property with bound 1 is equivalent to the question of whether there is an extremally disconnected compact Hausdorff space $E$ which is metrizable. (The following final section of course shows that the adjective "uniform" may be omitted.) By an

${ }^{3}$ A separable space $L$, such as $(c)$ or $\left(c_{0}\right)$, which has the separable extension property, has the property numbered (3) above. Also any transformation from an arbitrary separable space $M$ to such an $L$ has a continuous extension, with range in $L$.

The following modified property implies, but is not equivalent to, properties (1), (2), (3): (4) for each isomorphism $g$ of a subspace of $L$ onto a subspace of $L$, either $g$ or $g^{-1}$ has a continuous one-to-one linear extension to all of $L$, with range in $L$. 
Urysohn theorem, $E$ is metrizable if and only if it is separable. ${ }^{4}$

4. Proof of Theorem 1. We require the following lemma.

LEMmA. If two Banach superspaces $B_{1}, B_{2}$ of a Banach space $B$ have only $B$ in common, and if there exist continuous projections $p_{1}, p_{2}$ of respectively $B_{1}, B_{2}$ onto $B$, then there exists a Banach superspace $C$ which has $B_{1}$ and $B_{2}$ as subspaces.

Proof. If $B_{1}^{\prime}, B_{2}^{\prime}$ are the respective closed complements of $B$ in $B_{1}, B_{2}$ which are the null spaces of $p_{1}, p_{2}$, consider the Cartesian product $B \times B_{1}^{\prime} \times B_{2}^{\prime}$. With the usual definitions of addition and scalar multiplication, this Cartesian product is a linear space which has $B_{1}$ and $B_{2}$ as subspaces. Define a norm in $B \times B_{1}^{\prime} \times B_{2}^{\prime}$ as follows: $\left\|\left(\xi, \xi_{1}^{\prime}, \xi_{2}^{\prime}\right)\right\|=\sup \left|\gamma\left(\xi, \xi_{1}^{\prime}, \xi_{2}^{\prime}\right)\right|$ over all linear functionals $\gamma$ on the Cartesian product which simultaneously satisfy $\left|\gamma\left(\xi+\xi_{1}^{\prime}\right)\right|$ $\leqq\left\|\xi+\xi_{1}^{\prime}\right\|_{1}$ in $B_{1}$ and $\left|\gamma\left(\xi+\xi_{2}^{\prime}\right)\right| \leqq\left\|\xi+\xi_{2}^{\prime}\right\|_{2}$ in $B_{2}$, where $\xi \in B$, $\xi_{1}^{\prime} \in B_{1}^{\prime}, \xi_{2}^{\prime} \in B_{2}^{\prime}$. Then in $B_{1},\left\|\xi+\xi_{1}^{\prime}\right\|=\left\|\xi+\xi_{1}^{\prime}\right\|_{1}$, and in $B_{2},\left\|\xi+\xi_{2}^{\prime}\right\|$ $=\left\|\xi+\xi_{2}^{\prime}\right\|_{2}$, where \|\|$_{1},\|\|_{2}$ respectively are the norms of $B_{1}, B_{2}$. The required Banach space $C$ is the completion of $B \times B_{1}^{\prime} \times B_{2}^{\prime}$ with the norm \|\| .

Proceeding with the proof of the theorem, assume that $B$ has the projection property, but not the uniform projection property. Then there exist a sequence of superspaces $B_{1}, B_{2}, B_{3}, \cdots$, with minimal bounds for projections onto $B$ of $K_{1}, K_{2}, K_{3}, \cdots$, with $K_{1}<K_{2}$ $<K_{3}<\cdots$, and $K_{n} \rightarrow \infty$. We may consider $B_{1}, B_{2}, \cdots$ to be spaces which have only $B$ in common. Using the Lemma repeatedly, we define a sequence of superspaces $C_{1}, C_{2}, \cdots$, with $C_{1} \subset C_{2} \subset C_{3} \subset \cdots$, as follows. Space $C_{1}=B_{1} ; C_{2}$ is the common superspace of $C_{1}$ and $B_{2}$; $C_{3}$ is the common superspace of $C_{2}$ and $B_{3} ; \cdots$. Any projection of $C_{n}$ onto $B$ in particular is a projection of $B_{n}$ onto $B$; therefore the minimum bound for projections of $C_{n}$ onto $B$ is greater than or equal to $K_{n}$.

The union $C_{1} \cup C_{2} \cup C_{3} \cup \ldots$ is a linear space, if addition $\xi+\eta$ and scalar multiplication $h \xi$ are defined to coincide with addition and

\footnotetext{
4 Added note: It is shown in [16] that $C(E)$ is separable if and only if $E$ is a finite set. Therefore if an infinite-dimensional $B$ is separable and has the extension property, necessarily the bound $K$ is greater than 1 , and $B$ is not isomorphic with a space $C(E)$. Is such a $B$ always isometric with a complemented subspace of a space $C(E)$ ? Yes, for the following reasons. As in [16], $(m) \subset C(E)$ for every infinite $E$. An isometric imbedding of $(C)$ in $(m)$ is given in [11], and $(C)$ is a universal imbedding space for separable spaces. Consider the isometry $g$ of $B_{1} C(C) C(m)$ onto $B$. Since $B$ has the extension property, $g$ has a continuous extension to $(m)$, and $B_{1}$ is therefore complemented in $(m)$, also in every $C(E)$ since $(m)$ has the projection property.
} 
scalar multiplication in the space $C_{j}$ which contains $\xi, \eta$. Define a norm in the union by $\|\xi\|=\|\xi\|_{n}$, where $\|\xi\|_{n}$ is the norm in the first $C_{n}$ which contains $\xi$. Then contrary to assumption, the completion of the union is a Banach superspace of $B$ which cannot have a continuous projection onto $B$. Therefore as was asserted, the projection property implies the uniform projection property. ${ }^{5}$

Following is a further delimitation of the class of infinite-dimensional, separable Banach spaces $B$ which have the projection property. If $p$ is a continuous projection of $(m)$ onto $B C(m)$, and $g$ is an isometry of $B C(m)$ to $B C C$, where $C$ is an arbitrary Banach superspace of $B$, then $a=g p$ is a IIA transformation on $(m)$, with $R(a)=B C C$. Since $(m)$ has the extension property, there is a continuous extension $x$ of $g^{-1}$, and then $q=a x$ is a continuous projection of $C$ onto $B$. As a consequence of this, of Theorem 1, of result (12) on p. 96 of [18], and of Theorem 2 of [16], the space $B$ neither can be reflexive, nor possess an unconditional basis. Space $(C)$ qualifies [18, pp. 73-77], but it is not a space $B$. The results of [17] suggest that perhaps also all the successive conjugate spaces $B^{*}, B^{* *}, \cdots$ must be separable.

\section{REFERENCES}

1. F. Bohnenblust, Convex regions and projections in Minkowski spaces, Ann. of Math. (2) 39 (1938), 301-308.

2. R. H. Bruck, Survey of binary systems, Ergebnisse der Mathematik, Vol. 20, Springer, Berlin, 1958.

3. J. Dixmier, Les algèbres d'opérateurs dans l'espace Hilbertien (Algèbres de von Neumann), Cahiers scientifiques, Fas. XXV, Gauthier-Villars, Paris, 1957.

4. D. B. Goodner, Projections in normed linear spaces, Trans. Amer. Math. Soc. 69 (1950), 89-108.

5. B. Grünbaum, Extensions, retractions, and projections; Projection constants, Technical Notes Nos. 2; 6, Contract AF 61(052)-04, Hebrew University, Jerusalem, 1958.

6. J. L. Kelley, Banach spaces with the extension property, Trans. Amer. Math. Soc. 72 (1952), 323-326.

7. R. D. McWilliams, On projections of separable subspaces of $(m)$ onto (c), Proc. Amer. Math. Soc. 10 (1959), 872-876.

5 Added in proof. In a letter to the author, Professor Mahlon Day points out that the proof of Theorem 1 can be shortened by imbedding $B$ in the space of real bounded functions on the unit ball of the conjugate space $B^{*}$, and using 9 (d), p. 94 of [18]:

"If $U$ is the unit ball of $B^{*}$ (or a large enough subset of it), then there is the evaluation isometry $f$ of $B$ into $m(U)$. By 9 (d) there is a $g$ of some norm $k$ such that $g \circ f=i_{B}$. If $F$ is a linear isometry of $B$ into some $C$, then, by 9 a (Phillips), and $9(\mathrm{~d})$, there is a $G$ of norm 1 such that $G \circ\left(F \circ f^{-1}\right)=i_{f B}$. Then $[g \circ G] \circ F=i_{B}$ and $\|g \circ G\|$ $=k .9(\mathrm{~d})$ says $B$ has $P_{k .}$. 
8. L. Nachbin, $A$ theorem of the Hahn-Banach type for linear transformations, Trans. Amer. Math. Soc. 68 (1950), 28-46.

9. J. von Neumann (I. Halperin, Editor), Continuous geometry, Princeton Mathematical Series, Vol. 25, Princeton Univ. Press, Princeton, N. J., 1960.

10. R. S. Phillips, On linear transformations, Trans. Amer. Math. Soc. 48 (1940), 516-541.

11. A. Sobczyk, Projection of the space $(m)$ on its subspace ( $\left.c_{0}\right)$, Bull. Amer. Math. Soc. 47 (1941), 938-947.

12. - Projections in Minkowski and Banach spaces, Duke Math. J. 8 (1941), $78-106$.

13. - On the extension of linear transformations, Trans. Amer. Math. Soc. 55 (1944), 153-169.

14. B. Yood, Transformations between Banach spaces in the uniform topology, Ann. of Math. (2) 50 (1949), 486-503.

15. J. L. Kelley, General topology, Van Nostrand, New York, 1955.

16. R. C. James, Projections in the space (m), Proc. Amer. Math. Soc. 6 (1955), 899-902.

17. - Bases and reflexivity of Banach spaces, Ann. of Math. (2) 52 (1950), 518-527.

18. M. M. Day, Normed linear spaces, Ergebnisse der Mathematik, Vol. 21, Springer, Berlin, 1958.

19. D. B. Goodner, Separable spaces with the extension property, J. London Math. Soc. 35 (1960), 239-240.

20. N. Dunford and J. T. Schwartz, Linear operators, Part I, Interscience Publishers, New York, 1958.

University of Miami 